\title{
A Toddler With Pain Abdomen and Pica: The 'Concrete' Evidence!
}

\begin{abstract}
30-month-old boy presented with intermittent pain abdomen of three days duration. There was no fever, vomiting, diarrhea, dysuria or constipation. In between the episodes of pain, the child was comfortable. Vital signs and systemic examination, including per rectal examination, was normal. A plain $X$-ray abdomen in erect posture revealed multiple, radio-opaque shadows in the small intestine, colonic and the rectal area. The opacities were of different size and shape but of uniform density (Fig. 1). On further querying, mother admitted that the child has a habit of scrapping the old wall and eating small pieces of loose concrete material that used to fall off! The child was given liberal amount of oral fluids, soft diet, oral lactulose after hospital admission. After passage of stools thrice, on day 3, repeat $X$-ray abdomen showed complete clearance of the shadows. He had mild microcytic hypochromic anemia. Peripheral blood smear did not show any basophilic stippling. Blood lead assay could not be carried out. Parents were counselled regarding Pica and the provided behavioral modification advice. Child has been under regular follow up since then and is thriving well.
\end{abstract}

In our case, the morphology of opaque shadows along with the history given by mother helped in a 'concrete' diagnosis! As seen in this case, during follow up, such clear corroborative diagnosis can motivate the parents to ensure all the preventive steps to avoid consumption of such materials by the child again.

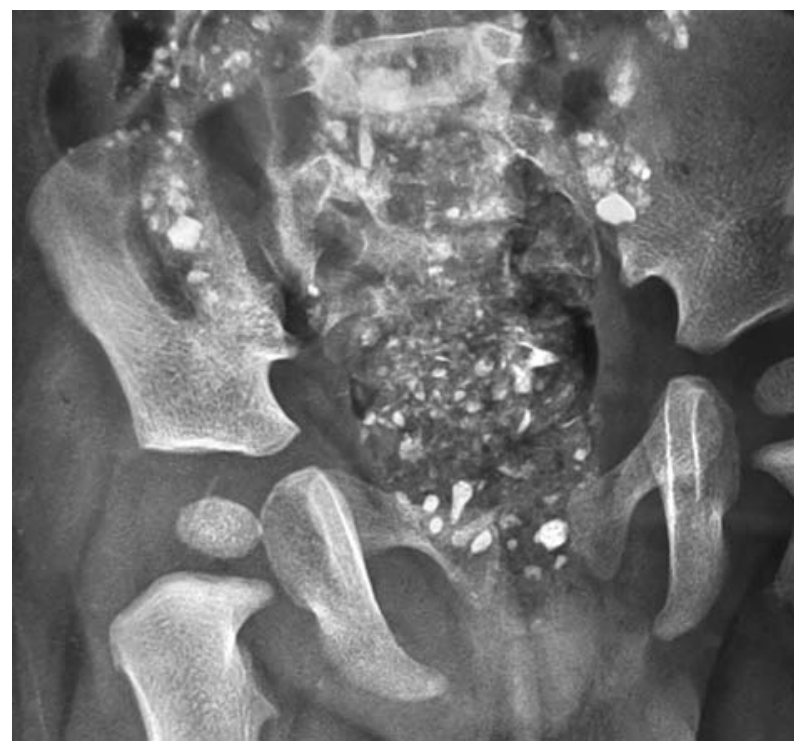

Fig. 1 Multiple opacities of varying shapes and sizes but of uniform density $X$-ray abdomen.

We share the typical $X$-ray picture to remind the readers of this common condition which is frequently overlooked.

\author{
K M ADHIKARI" AND GT SHAFIAHAMAD \\ Department of Pediatrics \\ INHS Asvini \\ Colaba, Mumbai, Maharashtra, India. \\ *kmadhikari@gmail.com
}

\section{Bronchial Dieulafoy Disease with Recurrent Life-threatening Hemoptysis}

Bronchial Dieulafoy's disease (BDD) denotes the presence of a vascular anomaly characterized by dilated, tortuous arteries in the bronchial submucosa, causing massive hemoptysis [1]. We present a child with life-threatening recurrent massive hemoptysis due to this disorder.

A 15-year-old boy, previously normal, had fainted after effortlessly expelling about $400 \mathrm{~mL}$ of blood and was intubated for airway protection. His hematological workup and coagulation and rheumatologic profile were normal. Upper gastrointestinal endoscopy demonstrated erosive gastritis, and he was treated for $H$. pylori. Ten days after discharge, he had an episode of drowsiness following a bout of cough with massive expectoration of blood. He was rushed to the emergency, where he was intubated, and received two units of packed cells.
Computed tomography of chest and pulmonary angiography showed patchy haziness in right lower lobe with no evidence of pulmonary embolism. He was referred to our hospital where he had a bout of massive hemoptysis and became limp. He was resuscitated, intubated, shifted to the pediatric intensive care unit and again required blood transfusion. Bronchoscopy revealed blood clots in right lower lobe. Broncho-alveolar lavage was negative for tuberculosis and fungal infections.

The clinical sequence of repeated massive life-threatening hemoptysis and blood clot on bronchoscopy was suggestive of bronchial artery pathology, possibly bronchial Dieulafoy disease. Selective aortography was done through right common femoral artery, a 4F cobra catheter was advanced into descending thoracic aorta. Selective embolization of hypertrophic tortuous right bronchial artery was performed using poly-vinyl alcohol (PVA) particles of 300-500 $\mu \mathrm{m}$ with complete disappearance of the abnormal right bronchial artery [2]. After embolization, the boy had no further hemoptysis and was doing well on follow-up after 6 months. 


\section{CORRESPONDENCE}

The most important step in situation of massive blood loss through the mouth is to first establish that the child is indeed experiencing hemoptysis. The boy was initially treated as hematemesis, and differentiating hemoptysis from hematemesis is critical, as treatment strategies differ markedly [3]. Definition of massive hemoptysis has not been completely agreed upon and vary between 200 and $600 \mathrm{~mL}$ as a cut-off, ideally, a volume $>200 \mathrm{~mL}$ warrants immediate investigation [4]. Although massive hemoptysis is relatively uncommon, mortality rates as high as $75 \%$ been reported. The common cause of death is asphyxia secondary to aspiration rather than blood volume loss. Selective bronchial artery embolization is an effective nonsurgical technique for immediate control of massive hemoptysis with a success rate around of 77 - 94\% [5]. Bronchial dieulafoy disease is rare disease but known to cause massive hemoptysis, where bronchial artery embolization should be considered as the first-line treatment.
Acknowledgements: Dr R Ravikumar and Dr. Pritam Chatterjee for surgical intervention.

D ViJAYASEkARAN* AND Somu Sivabalan Kanchi Kamakoti CHILDS Trust Hospital Chennai, India. *vijsekar@hotmail.com

\section{REFERENCES}

1. Qian X, Du Q, Wei N, Wang M, Wang H, Tang Y. Bronchial Dieulafoy disease: A retrospective analysis of 73 cases. BMC Pulm Med. 2019;19:104.

2. Sopko DR, Smith TP. Bronchial artery embolization for hemoptysis. Semin Intervent Radiol. 2011; 28:48-62.

3. Batra PS, Holinger LD. Etiology and management of pediatric hemoptysis. Arch Otolaryngol Head Neck Surg. 2001;127:377-82.

4. Ibrahim W. H. Massive hemoptysis: The definition should be revised. Euro Resp J. 2008;32:1131-32.

5. Lopez JK, Lee HY. Bronchial artery embolization for treatment of life-threatening hemoptysis. Semin Intervent Radiol. 2006;23:223-29.

\section{The Incompleteness of Incomplete Kawasaki Disease: A Customized Definition Is Needed for Indian Children}

We read with interest the Indian Academy of Pediatrics position paper on Kawasaki disease in the journal [1]. It is indeed timely that this statement has come out amidst the coronavirus disease (COVID-19) pandemic and associated multisystem inflammatory syndrome in children. The authors have aimed to present this paper as a practice guideline specific to resource constrained setting like ours. In this context, we have the following comments:

In describing the definition for incomplete $\mathrm{KD}$, the authors have presented the diagnostic approach, which is largely adapted from American Heart Association (AHA) scientific statement on Kawasaki disease [2]. While AHA algorithm considers evaluation for incomplete KD in children with fever $\geq 5$ days and 2 or 3 compatible clinical criteria, the algorithm by Shenoy, et al. [1] triggers KD evaluation if fever $\geq 5$ days is accompanied by less than four compatible clinical features. Although these two statements appear similar, this approach loses specificity by including children who present with fever and just one compatible clinical feature. Individually, the clinical features like rash, lymphadenopathy, conjunctival injection, oral or extremity changes are nonspecific and may occur with various childhood infections in India. This approach risks huge number of children with underlying infections being referred for echocardio-graphic evaluation.

Treatment with intravenous immunoglobulin is recommended if 3 of the 5 laboratory features (anemia for age, platelet $\geq 450 \times 10^{9} / \mathrm{L}$, albumin $<3 \mathrm{~g} / \mathrm{dL}$, elevated alanine aminotransferase, leucocyte count $\geq 15 \times 10^{9} / \mathrm{L}$, urine $>10$ $\mathrm{WBC} / \mathrm{hpf}$ ) are met in a child lacking echocardiographic abnormalities. Compared to Western cohorts, these criteria should be carefully defined in a low- and middle-income setting like India, with a high prevalence of iron deficiency anemia [3] and associated thrombocytosis (present in up to a quarter of those with iron deficiency) [4]. Iron deficiency when associated with infection accounted for more than half of all cases of reactive thrombocytosis in Indian children [5]. Given these findings, the current definition is likely to overestimate the burden of incomplete $\mathrm{KD}$ in Indian children, risking increased cost and potentially delaying the diagnosis of underlying infections. For example, as per the algorithm, a child with undifferentiated fever $\geq 5$ days due to measles or a rickettsial infection that has a rash, iron deficiency anemia (and associated thrombo-cytosis) and hypoalbuminemia (negative acute phase reactant) would be treated for Kawasaki disease even if the echocardiogram is normal. In the absence of a 'gold standard' for diagnosis, we believe that grading recommendations based on available quality of evidence may be more useful for the readers to make informed decisions [6].

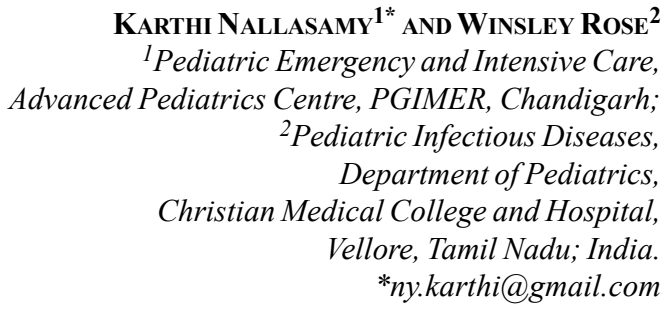

\section{REFERENCES}

1. Shenoy B, Singh S, Ahmed MZ, et al. Indian Academy of Pediatrics Position Paper on Kawasaki Disease. Indian Pediatr. 2020;57:1040-48.

2. McCrindle BW, Rowley AH, Newburger JW, et al. Diagnosis, Treatment, and Long-Term Management of Kawasaki Disease: A 